\title{
Planes de desarrollo y ordenamiento territorial. Estudio de caso: Gobierno Autónomo Descentralizado municipal de Portoviejo
} \author{
Government of Portoviejo \\ "Roxana Jaqueline Arteaga Demera" $" *$ \\ "Norberto Pelegrín Entenza"2 \\ "Susetty Gómez García”3
}

Development plans and land use planning. Case study: Municipal Decentralized Autonomous

\section{Resumen}

La dependencia fiscal de los gobiernos locales ha sido motivo de inmovilización de la gestión, lo que ha limitado el despliegue de la inversión pública en función de las prioridades territoriales, todo por la invisibilidad otorgada a los instrumentos de planificación local. La investigación presenta un caso de estudio en el Gobierno Autónomo Descentralizado municipal del cantón Portoviejo, cuyo objetivo fue evaluar la efectividad de su sistema de planificación, reflejada en la calidad de su Plan de Desarrollo y Ordenamiento Territorial. Se desarrolló un estudio explicativo, con un enfoque mixto, no experimental, basado en el análisis documental, la observación, la aplicación de encuestas y entrevistas; el estudio de caso y la estadística descriptiva, a partir de una matriz de indicadores que fueron evaluados con estos instrumentos. Como resultado, se revelan algunos avances positivos en el sistema de planificación de este gobierno local, que bien podrían orientar a otros niveles de gobierno, además, se identifican las limitaciones existentes, cuya incidencia es principalmente a nivel nacional. Es necesario repensar la planificación local con una visión de sostenibilidad y derecho a la ciudad, con una gobernanza responsable y una participación real de todos los actores involucrados en el desarrollo local.

\begin{abstract}
The fiscal dependence of local governments has been a cause for the immobilization of management, which has limited the deployment of public investment based on territorial priorities, all due to the invisibility given to local planning instruments. The research presents a case study in the Municipal Autonomous Decentralized Government of the Portoviejo canton, whose objective was to evaluate the effectiveness of its planning system, reflected in the quality of its Development Plan and Territorial Organization. An explanatory study was developed, with a mixed, non-experimental approach, based on documentary analysis, observation, the application of surveys and interviews; the case study and descriptive statistics, based on a matrix of indicators that were evaluated with these instruments. As a result, some positive advances are revealed in the planning system of this local government, which could well guide other levels of government, in addition, existing limitations are identified, whose incidence is mainly at the national level. It is necessary to rethink local planning with a vision of sustainability and the right to the city, with responsible governance and real participation of all the actors involved in local development.
\end{abstract}

\section{Palabras clave/Keywords}

Desarrollo; planificación; ordenamiento territorial; gobierno local/Development; planning; territorial ordering; local government

*Dirección para correspondencia: rarteagademera@yahoo.es

Artículo recibido el $19-11-2020$ Artículo aceptado el 13-12-2020 Artículo publicado el $30-12-2020$

Conflicto de intereses no declarado.

Fundada 2016 Unidad de Cooperación Universitaria de la Universidad Técnica de Manabí, Ecuador.

\footnotetext{
${ }^{1}$ Estudiante de la maestría de investigación en Desarrollo Local, Instituto de Posgrado, Universidad Técnica de Manabí, Ingeniera en Auditoría, Portoviejo, Ecuador, rarteagademera@yahoo.es, https://orcid.org/0000-0002-2444-4074

${ }^{2}$ Universidad Técnica de Manabí, Docente e investigador, Doctor, Portoviejo, Ecuador, norbepelegrin@ gmail.com, https://orcid.org/0000-0001-7445-7423

${ }^{3}$ Universidad Técnica de Manabí, Docente e investigador, Magister, Portoviejo, Ecuador, susettylore@ hotmail.com, https://orcid.org/0000-0001-8593-1979
} 


\section{Introducción}

Los planes de desarrollo y ordenamiento territorial (PDOT), revisten una gran importancia y significación, pues según el Código Orgánico de Planificación y Finanzas Públicas (COPFP), contienen las directrices principales que permiten la gestión concertada y articulada del territorio. $\mathrm{Su}$ objetivo es ordenar, compatibilizar y armonizar las decisiones estratégicas de desarrollo de cada territorio. En los tres niveles de gobierno, provincial, cantonal y parroquial se deben articular los planes entre sí, considerando, lo normado para la regulación del uso y ocupación del suelo.

Se elaboran siguiendo un proceso de al menos tres fases, que se representan en la figura 1, que incluye diagnóstico, propuesta y modelo de gestión.

Diagnóstico Estratégico - Modelo Territorial Actual
$\begin{aligned} & \text { El diagnóstico es de carácter estratégico. Muestra la situación que } \\ & \text { atraviesa un territorio y su población e identifica las potencialidades, } \\ & \text { deficiencias o carencias. }\end{aligned}$
Propuesta - Modelo Territorial Deseado
$\begin{aligned} & \text { Son las decisiones estratégicas y territoriales que deben } \\ & \text { implementarse para alcanzar la visión de desarrollo en el corto, } \\ & \text { mediano y largo plazo. }\end{aligned}$
$\begin{aligned} & \text { Modelo de Gestión - Ejecución del PDOT } \\ & \text { Conjunto de estrategias y procesos que implementa el GAD para } \\ & \text { administrar su territorio. }\end{aligned}$

Figura 1. Contenidos mínimos del PDOT.

Fuente: Guía para la formulación/actualización del Plan de Desarrollo y Ordenamiento Territorial (PDOT) Cantonal (Secretaría Técnica Planifica Ecuador, 2019).

La fase de diagnóstico es de vital importancia dado que permite identificar la situación actual del territorio, sin embargo, requiere un cambio de enfoque para mejorar su construcción. A juicio de los autores, es necesario hacer las siguientes reflexiones para fortalecer los resultados que se esperan de esta fase:

- Los componentes sobre los cuales se desarrolla el diagnóstico deben estar atados a la visión de desarrollo que tiene el gobierno local.

- Los sistemas locales de información se encuentran debilitados, se requiere que el Estado genere información local.

- La información del diagnóstico, necesita tener un carácter estratégico. La conexión entre el diagnóstico y la propuesta es vital.

En la fase de propuesta, el objetivo debe ser planificar y ordenar el territorio, fortalecer las potencialidades y minimizar las problemáticas a través de programas, proyectos, planes y procesos.

La fase de modelo de gestión obliga a considerar el vínculo entre los objetivos estratégicos, la política pública y las estrategias de articulación, por esa razón deben estar claramente definidos en el plan, para garantizar la funcionalidad del modelo.

Este instrumento, debe convertirse en la hoja de ruta de la gestión pública de los gobiernos locales, además de ser el sustento de la acción política de sus gobernantes. La técnica, orientada al pensamiento del desarrollo humano sostenible, para devolver a las personas el derecho a la ciudad, es lo que permitirá el verdadero desarrollo de una localidad.

Ecuador, ha dado un paso importante al referirnos a la construcción y aprobación de un marco constitucional que busca cambios en el modelo de pensar y actuar sobre el desarrollo, y sitúa el buen vivir como un nuevo paradigma. En ese nuevo escenario, la planificación del desarrollo es fundamental para la organización, dirección, y control de la gestión pública, la garantía de los derechos ciudadanos, el fomento de la participación ciudadana y el control social, la construcción de un sistema económico social, solidario y sostenible, el equilibrio territorial, y propiciar a través de la política pública, la convivencia armónica y el desarrollo humano.

La vigente Constitución del Ecuador (Asamblea Nacional Constituyente de Ecuador, 2008), en el título VI, capítulo II, reconoce la acción de la planificación y dentro de ella, garantiza los derechos de la población. La planificación nacional está considerada como un deber del Estado; el Código Orgánico de Planificación y Finanzas Públicas se refiere al fortalecimiento de procesos participativos y de planificación para el desarrollo (Asamblea Nacional del Ecuador, 2010b); el Código Orgánico de Organización Territorial, Autonomía y Descentralización, destaca la necesidad de incentivar la participación ciudadana en el desarrollo territorial (Asamblea Nacional del Ecuador, 2010a), desde esta visión, la construcción del territorio deseado (planificación prospectiva), es el producto de la visión concertada de quienes integran una población.

La solvencia institucional de los gobiernos locales es casi siempre una de las causas de la inmovilización para visionar y ejecutar una propuesta de desarrollo integral de sus territorios, por lo que es necesario desarrollar procesos disruptivos basados en metas y orientados a resultados de corto, mediano y largo plazo, que superen la gestión de la administración pública tradicional y emprendan liderazgos locales de los gobiernos autónomos.

A nivel local, el desafío de la formulación de los Planes de Desarrollo y Ordenamiento Territorial sigue estando en el debate público, se trata de la consolidación del desarrollo y del ordenamiento de los territorios, que plantee acciones tendientes a abordar problemáticas locales muy fuertes, con miras a transformar las condiciones de vida de las personas, a generar inclusión y movilidad económica equitativa en la sociedad.

Desarrollar un proceso de planificación real, responsable, coherente, y al que se le asignen recursos, aportará a la construcción de un modelo de Estado menos centralista, que enfatice en una visión de desarrollo sostenible del territorio, donde la territorialización de la política pública enfocada en la reducción de brechas de inequidad y desigualdad, y la satisfacción de necesidades básicas de la población, sean una prioridad.

La actuación de las dinámicas locales, previstas en los planes de desarrollo, debe aportar a la consolidación de un nuevo modo de acumulación y redistribución, donde la articulación de las intervenciones públicas en todos los niveles de gobierno reafirme el rol redistributivo del Estado.

Estos desafíos exigen al Estado Central y a los Gobiernos Autónomos Descentralizados la aplicación de mecanismos de coordinación sectorial y local, que contribuyan en la construcción del modelo territorial deseado.

Si bien las bases normativas, jurídicas y legales están determinadas por el órgano máximo de regulación que es la Constitución de la República; se comparten los criterios de Alburquerque (2004) cuando expresa que, en los niveles territoriales existe una elevada disparidad en el desarrollo de las localidades, y un débil sistema de planificación local, entendiéndose a la planificación como lo menciona Alonso \& Otero (2020), como una gestión integral que prioriza programas, proyectos, planes, y procesos, que de concretarse ya sea con fuentes de financiamiento internas o externas; podría mejorar las condiciones de vida de la población.

Frente a las inequidades estructurales determinantes para el desarrollo de un territorio y de la población en su conjunto, reafirmando que por mandato constitucional existen los marcos legales, jurídicos, administrativos y de participación ciudadana que norman y determinan sistemas y procesos orientadores del desarrollo local, y de la acción pública sectorial en los territorios, surgen las interrogantes ¿qué causas

Planes de desarrollo y ordenamiento territorial. Caso de estudio: Gobierno Autónomo Descentralizado municipal de Portoviejo

Arteaga Demera, Pelegrín Entenza, Gómez García 
provocan las brechas existentes entre el estado actual y el estado deseado? ¿qué falta por hacer a los gobiernos locales y a la sociedad?

La investigación, muestra un estudio de caso, desarrollado en el Gobierno Autónomo Descentralizado municipal de Portoviejo, un cantón capital de provincia, cuya población proyectada al 2020 por el Instituto Nacional de Estadísticas y Censos INEC es de 321.800, lo que significa que es el cantón mayormente poblado de la provincia, a más de ser catalogado por la Cátedra UNESCO como ciudad intermedia (menos de 1 millón de habitantes y acoger a más del $50 \%$ de la población en la zona urbana). Por sus características territoriales endógenas ejerce una influencia notable sobre el resto de los cantones.

El modelo de administración del gobierno municipal se basa en una gestión por procesos enfocada en resultados, que ha sido referencia local y nacional, a tal punto que en agosto del año 2020 recibió el reconocimiento del programa Modelo Ecuatoriano de Calidad Excelencia, nivel comprometido, del Ministerio de Trabajo; galardón que se le ha asignado al primer y único municipio del país.

Con el afán de contribuir en el fortalecimiento de la gestión local a través de los Planes de Desarrollo y Ordenamiento Territorial PDOT, se desarrolla este caso de estudio. El objetivo del artículo fue evaluar la efectividad del sistema de planificación del Gobierno Autónomo Descentralizado municipal del cantón Portoviejo, reflejada en la calidad de su Plan de Desarrollo y Ordenamiento Territorial.

\subsection{Marco teórico}

La palabra desarrollo usualmente ha sido relacionada a los avances y progresos en el campo económico. La Real Academia Española traduce al desarrollo como la "evolución progresiva de una economía hacia mejores niveles de vida", mientras que, cuando se refiere a las personas, se define como progreso, bienestar, modernización, crecimiento económico, social, cultural o político. En las ciencias sociales y la política, el desarrollo alude a un amplio abanico de asuntos académicos y prácticos (RAE, 2017).

Gudynas (2018) afirma que el sentido convencional del desarrollo, y en particular la llamada "economía del desarrollo", se popularizó inmediatamente después de la segunda guerra mundial, como respuesta práctica a la pobreza y a la distribución de la riqueza. Se distinguieron por un lado los países desarrollados, y por el otro, las naciones subdesarrolladas, entre ellas América Latina.

Poco tiempo después comenzaron a aparecer los primeros cuestionamientos y debates para separar "desarrollo" de "crecimiento", es decir los aspectos cualitativos de los cuantitativos, pues era necesario involucrar aspectos ambientales sociales y culturales, y no solamente económicos, en ese sentido, Sen (2000) propone que el desarrollo requiere la eliminación de importantes fuentes de la ausencia de libertad como son la pobreza y la tiranía, oportunidades económicas escasas y privaciones sociales sistemáticas, falta de servicios públicos e intolerancia de estados represivos.

El Banco Mundial (2020) menciona que alrededor del 55\% de la población mundial, esto es 4200 millones de habitantes, viven en ciudades. Se cree además que esta tendencia continuará, y que, en el 2050, la población urbana se duplicará; 7 de cada 10 personas vivirán en ciudades.

En ese sentido, en territorios urbanizados, se necesitarán proyectos colectivos de ciudad que no se basen tan solo en el desarrollo de infraestructuras físicas y en el consumo descabellado de recursos, sino que desde los gobiernos locales se planifique ese crecimiento poblacional garantizando el acceso a servicios básicos.
Una de las teorías más recientes y que a juicio de los autores tiene un enfoque desafiante y acertado, es la del desarrollo sostenible, al poner de manifiesto la prioridad de la satisfacción de necesidades presentes y futuras, garantizando el equilibrio entre el crecimiento económico, el cuidado del medio ambiente y el bienestar social, lo que resulta muy complejo, sobre todo en países con economía de capital.

El desarrollo sostenible, es un enfoque predominante en la actualidad a nivel mundial, que hace énfasis a la degradación progresiva del medio ambiente como uno de los grandes problemas actuales, y que tiene que ver con el comportamiento humano y su capacidad de transformar el ambiente que le rodea, en sentido se coincide con los postulados de Gómez (2020) cuando plantea la necesidad de generar condiciones políticas para el desarrollo de este modo de producción.

En ese sentido, CEPAL (2018) en su publicación Agenda 2030 y los Objetivos de Desarrollo Sostenible los define como una oportunidad para construir una agenda política para la sostenibilidad económica, social y ambiental del mundo.

Así también UNESCO (2017), promueve la educación para el desarrollo sostenible (EDS), que tiene que ver con la generación de condiciones que provoquen un cambio de actitudes y conductas que nos conduzcan a sociedades más sostenibles; para lograrlo se necesita un cambio en los estilos de vida y en la forma de pensar y actuar, y es allí donde el sistema educativo cumple un rol importante introduciendo nuevas pedagogías.

Un aporte que no puede quedar de lado es la teoría del "desarrollo a escala humana" promovido por el economista chileno Manfred MaxNeef, que se basa en tres postulados centrales: el desarrollo se enfoca en las personas y no en los objetos, distingue satisfactores de necesidades, y la pobreza es un concepto plural que depende de las necesidades insatisfechas (Max-Neef et al., 2010). A partir de este acertadísimo postulado es que se desarrollan ciudades para la gente como lo dice Gehl (2014).

Una reivindicación en el sistema de planificación territorial es alcanzar espacios al servicio de la gente y no que la gente se ubique a disposición de la ciudad o del mercado, sin embargo, esto aún se debate entre las políticas públicas y acciones organizativas de las propias personas.

El desarrollo sostenible de la mano con el desarrollo a escala humana, nos hacen pensar desde vientos esperanzadores, sobre todo porque se colocan en el debate público, de sectores académicos, de gobierno, de organizaciones sociales, la concepción del desarrollo no como un asunto menor, sino como un factor determinante para la vida y progreso de los pueblos; paralelo a ello se requieren instrumentos sobre los cuales se asiente esa visión de desarrollo, y es el rol que deben cumplir los planes de desarrollo y ordenamiento territorial para propiciar el desarrollo local.

Blanco (2003) menciona que el desarrollo de los territorios no es sinónimo de desarrollo municipal, sino que se trata de un proceso impulsado desde abajo hacia arriba, apoyado en las capacidades endógenas de los territorios, como también lo menciona Quispe (2016), sin embargo Castellanos (2017), contrariamente refiere que desarrollo local es un tema del gobierno local, dado que implica lo administrativo y la gestión, aunque refiere también que no debe reducirse a ello, pues necesita procesos en un nivel más alto.

Se considera que es fundamental que este proceso este liderado principalmente por los gobiernos locales, en conjunto con la comunidad, las asociaciones o empresas locales, y de ser necesario algunas instituciones de apoyo presentes en el territorio, sin embargo, es 
fundamental la inclusión de actores externos que sean parte de la propuesta de desarrollo sin tener que limitarse a ello.

Por su parte CEPAL (2015) plantea algunas consideraciones importantes coincidentes con Riffo (2017):

- No todos los territorios están igualmente preparados para enfrentar los procesos de apertura y globalización.

- Hay un gran nivel de disparidad territorial que requiere distintos tipos de intervención.

- Las capacidades técnicas y liderazgos en las localidades son desiguales.

- La lógica del desarrollo integral y apoyo al desarrollo productivo no es un discurso generalizado en lo local.

Lo que aún sigue en debate, es la forma en que se construye el desarrollo local y el rol de la población, lo que no queda duda es que en la actualidad este tipo de desarrollo se caracteriza por su carácter autónomo, donde la fuerza de sus recursos y de las capacidades propias de sus habitantes controlarán el proceso a través de las iniciativas de desarrollo local. Estas decisiones sin duda pueden enriquecerse cuando se expanden al exterior, pues es necesaria la convergencia de varios territorios en procesos de desarrollo.

Alonso \& Otero (2020) plantea que el desarrollo local difiere de la política clásica de ordenación del territorio en que su objetivo no es la localización de inversiones en el espacio, sino del fomento de nuevas actividades productivas y empresariales desde planteamientos de desarrollo endógeno y sostenible en el territorio, lo que a juicio propio se considera un desacierto que la localización de inversiones en el espacio no sea una prioridad, dado que los recursos locales no son suficientes para generar condiciones de desarrollo en los territorios, no basta la generación de actividades productivas que ensanchen la oferta frente a una demanda que carece de capacidad adquisitiva, la economía de subsistencia no supera pobreza, al contrario engrandece los cinturones de precariedad, y a la hora de planificar programas y proyectos se debe tener claro el modelo de desarrollo que se pretende aplicar.

No cabe duda de que para que exista desarrollo local integral y sostenible deben generarse articulación público-privada que priorice las iniciativas locales. En la publicación de CONGOPE (2017) se señala que dentro del sector privado existen múltiples empresas locales cuya responsabilidad social corporativa está orientada no solo a la mitigación de aquellos aspectos negativos derivados de su actividad, sino a generar un valor añadido social que permita mejorar el bienestar del entorno en el que se encuadran.

Se coincide con lo señalado por los autores en tanto que la responsabilidad social del sector privado debe corresponder a las condiciones de mejora que tiene el propio sector por el giro de su negocio, pero hay que ser cuidadosos de no promover un Estado de bienestar, que tienda al paternalismo con los más "pobrecitos" de la localidad, ese enfoque además de ser excluyente es denigrante, pues se trata de generar equidad, a partir del derecho que tienen las personas a una vida digna, y al goce y disfrute del territorio, lo que se conoce como derecho a la ciudad; el desarrollo local por ende debe promover condiciones de justicia social.

El derecho a la ciudad no es una propuesta nueva (ONU, 2018), se trata de un concepto multidimensional y un movilizador social objeto de la acción gubernamental (Brenes et al., 2014). El término apareció en 1968 cuando el filósofo y geógrafo francés Henri Lefebvre tomó en cuenta el impacto negativo sufrido por las ciudades en los países de economía capitalista, con la conversión de la ciudad en una mercancía al servicio exclusivo de los intereses de la acumulación del capital. Este autor señala que, entre los derechos básicos de los ciudadanos, se debía incluir el derecho a vivir en territorios propicios a la convivencia y pluralidad de usos diversos, en los cuales los equipamientos públicos sean factores de desarrollo colectivo e individual (Molano, 2016).

La conceptualización del territorio es fundamental cuando se profundiza en los enfoques de la planificación, en ese sentido un enfoque interesante es el planteado por Gómez (2008) cuando coloca al territorio como un sistema construido socialmente en el que se articulan las actividades de la población sobre el medio físico, con un enfoque en la distribución del ingreso, dentro de la necesaria lucha contra la pobreza y la desigualdad como lo analiza Alburquerque (2004).

El territorio nos da constantemente la posibilidad de ser disruptivos y de reinventar, y a partir de esa filosofía, todas las ciudades que buscan sostenibilidad empiezan a construir su visión, como lo explica Gardner et al. (2016)

Históricamente, se ha concebido al "desarrollo" desde la planificación de la obra pública y no desde las personas, lo que impide entender a la planificación como una motivación de y para la transformación de las relaciones entre las personas, es allí donde la planificación estratégica del territorio se convierte en una herramienta para ganar, consolidar o alcanzar la competitividad requerida para la inserción económico-social (Castiella, 2016).

Güell (2019) frente a la planificación estratégica señala que la clave está en orientar a los objetivos hacia la competitividad para la mejora de la eficiencia de un sistema que busca resultados de calidad, y en ese sentido, existen metodologías muy innovadoras que pueden ayudar a las organizaciones en el desarrollo de sus planes.

Coincidencia que menciona Romero \& Vidal (2018) a más de añadir las metodologías innovadoras que se deben aplicar para el entendimiento de los objetivos de competitividad (resultados) como objetivos de mejora continua en la eficiencia.

La mejora continua, enfocada en la teoría de la calidad fue desarrollada por el norteamericano Edward Deming, quien plantea este ciclo como una herramienta basada en una serie de actividades para el mejoramiento, que inicia con un estudio de la situación actual, durante el cual se reúnen los datos que van a usarse en la formulación del plan para el mejoramiento; una vez que este plan ha sido terminado, es ejecutado.

Después de eso, se revisa la ejecución para ver si se han producido los mejoramientos anticipados. Si el experimento ha tenido éxito, se emprende una acción final, tal como la estandarización metodológica, para asegurar que los nuevos métodos se apliquen de forma continua para el mejoramiento sostenido (Colorado, 2009).

Se trata del conocido ciclo PHVA, que significan Planificar, Hacer, Verificar y Actuar que como lo menciona Gómez (2015) puede aplicarse a todos los procesos y al sistema de gestión de la calidad como un todo.

En Ecuador, con el afán de introducir a la administración pública en la cultura de la excelencia y de los principios del GCT (Gestión de la Calidad Total, TQM en sus siglas inglesas), se desarrolla el Modelo Ecuatoriano de Calidad y Excelencia alineado al ciclo PHVA, con el objetivo de impulsar a las instituciones públicas a su mejora permanente a fin de satisfacer cabalmente las necesidades y expectativas de la ciudadanía de acuerdo con resultados cuantificables (Ministerio de Trabajo, 2018a,b).

Bajo el enfoque de calidad, el planeamiento estratégico es un esfuerzo organizacional, definido y disciplinado, que busca que las instituciones definan con la mayor claridad posible sus acciones y sus objetivos de futuro (Andía, 2016).

En esa línea el Project Management Institute (PMI), advierte que los objetivos mal definidos constituyen un obstáculo para lograr una gestión orientada a resultados y para evaluar si los resultados han sido alcanzados, y bajo esa primicia sugiere que, al definir los objetivos, se eviten objetivos 
vagos o de interpretación dudosa, mientras más detallado sea el objetivo, mejor será su comprensión y mayores las probabilidades de que sea alcanzado. En ese sentido plantea que los objetivos deben ser: específicos, mesurables, alcanzables, realistas, y definidos en el tiempo (Siles \& Mondelo, 2018).

El proceso de prever el futuro es muy diferente de la planeación a largo plazo o la planificación prospectiva; el primero, a menudo, es simplemente la extrapolación de tendencias actuales. La prospectiva es más que tratar de anticipar el futuro y prepararse en forma apropiada; implica la convicción de que lo que hace la institución ahora puede influir en los aspectos del futuro y modificarlos.

En esa misma línea, es propicio mencionar que la dinámica de la actividad prospectiva ha generado una diferenciación muy amplia de prácticas en diferentes niveles y escalas de intervención (global, sectorial, institucional, territorial e interterritorial). Esto señala una agenda de temas a largo plazo para promover el cambio estructural en la región, donde se requiere que el Estado asuma un rol más activo y que la planificación desempeñe un papel crucial en el diseño y la implementación de políticas públicas para el desarrollo (Máttar \& Perrotti, 2014).

Los trabajos de prospectiva están adquiriendo creciente importancia en los países avanzados. Según Bitar (2016), su volumen ha aumentado sostenidamente, y se han multiplicado los estudios realizados por gobiernos, organismos internacionales, universidades o centros independientes.

Bajo esta tendencia de la planificación, el cantón Portoviejo desarrolla su planificación con una visión prospectiva: Plan Portoviejo 2035, que contiene el plan de uso y gestión de suelo (Asamblea Nacional del Ecuador, 2016, 2019); un instrumento regulatorio de notada importancia que supera las serias limitaciones y los impedimentos de los sistemas de planeamiento urbano que tienen nuestros países tanto para implementar políticas eficaces que prioricen y orienten la promoción, crecimiento y desarrollo urbano de las ciudades, como para regular suficientemente la participación del sector privado o la simple elaboración de planes y normas legales de las actividades urbanas, que en sentido amplio pueden llegar a condicionar el rol estatal en la planificación urbana y en la gestión de proyectos arquitectónicos, y pueden permitir encontrar recursos que financien ciertos programas de interés colectivo (Abramo et al., 2016).

Por otro lado, Noguera (2016) menciona que uno de los principales escollos a los que se enfrenta la planificación es la circunscripción de los planes a lo políticamente correcto, tanto en lo concerniente al resultado que el tomador de decisión prefiere esperar como aquello que sea compatible con la costumbre de planificación de gobierno, lo que pondría en riesgo la planificación estratégica y la visión prospectiva del desarrollo local y del ordenamiento del territorio.

\section{Materiales y Métodos}

La investigación devela los avances y limitaciones existentes en el sistema de planificación del Gobierno Autónomo Descentralizado Municipal del cantón Portoviejo, reflejada en la calidad de su plan de desarrollo y ordenamiento territorial. Es una investigación aplicada, un estudio de tipo explicativo al establecer relaciones causa-efecto entre las variables que inciden en la eficacia de los PDOT.

Según los datos utilizados, es de tipo mixta, es decir cuantitativacualitativa y no experimental; se basó fundamentalmente en el análisis documental, la observación, la aplicación de encuestas, y entrevistas; el estudio de caso y la estadística descriptiva. Se aplicó además la triangulación disciplinaria, metodológica, de datos, fuentes e investigadores.

Para la estructuración de la investigación, se desarrolló una matriz que contiene los principales indicadores a ser evaluados, para determinar las fortalezas y limitaciones que presenta el GAD Portoviejo en su sistema de planificación, como se muestra en la tabla 1.

Tabla 1.

Indicadores evaluados

\begin{tabular}{|c|c|c|c|}
\hline No. & Componente & Indicadores & $\begin{array}{l}\text { Niveles de } \\
\text { gestión }\end{array}$ \\
\hline 1 & $\begin{array}{l}\text { Planificación/ } \\
\text { Procesos }\end{array}$ & $\begin{array}{l}\text { Número de procesos levantados } \\
\text { para la ejecución y control del } \\
\text { PDOT }\end{array}$ & Estratégico \\
\hline 2 & $\begin{array}{l}\text { Planificación/ } \\
\text { Control }\end{array}$ & $\begin{array}{l}\text { Número de reportes sobre } \\
\text { seguimiento al cumplimiento de } \\
\text { metas }\end{array}$ & Operativo \\
\hline 3 & $\begin{array}{l}\text { Planificación/ } \\
\text { Control }\end{array}$ & $\begin{array}{l}\text { Número de reprogramación de las } \\
\text { metas del PDOT }\end{array}$ & Operativo \\
\hline 4 & $\begin{array}{l}\text { Planificación/ } \\
\text { Control }\end{array}$ & $\begin{array}{l}\text { Número de reuniones con las } \\
\text { áreas del GAD para informar } \\
\text { estado de las metas }\end{array}$ & Táctico \\
\hline 5 & $\begin{array}{l}\text { Planificación } \\
\text { estratégica }\end{array}$ & $\begin{array}{l}\text { Número de reportes de avances } \\
\text { de metas al ejecutivo local }\end{array}$ & Estratégico \\
\hline 6 & $\begin{array}{l}\text { Planificación } \\
\text { estratégica }\end{array}$ & $\begin{array}{l}\text { Número de reportes de avances } \\
\text { de metas al legislativo local }\end{array}$ & Estratégico \\
\hline 7 & $\begin{array}{l}\text { Planificación } \\
\text { estratégica }\end{array}$ & $\begin{array}{l}\text { Índice de prosperidad de } \\
\text { ciudades }\end{array}$ & Estratégico \\
\hline 8 & $\begin{array}{l}\text { Planificación } \\
\text { institucional }\end{array}$ & $\begin{array}{l}\text { Porcentaje de avance de los } \\
\text { objetivos estratégicos }\end{array}$ & Estratégico \\
\hline 9 & $\begin{array}{l}\text { Planificación } \\
\text { institucional }\end{array}$ & $\begin{array}{l}\text { Porcentaje de avance de los } \\
\text { programas }\end{array}$ & Táctico \\
\hline 10 & $\begin{array}{l}\text { Planificación } \\
\text { institucional }\end{array}$ & $\begin{array}{l}\text { Porcentaje de avance de los } \\
\text { proyectos y planes }\end{array}$ & Operativo \\
\hline 11 & $\begin{array}{l}\text { Planificación/ } \\
\text { Sostenibilidad } \\
\text { institucional }\end{array}$ & $\begin{array}{l}\text { Índice de capacidad de } \\
\text { endeudamiento }\end{array}$ & Táctico \\
\hline 12 & $\begin{array}{l}\text { Planificación/ } \\
\text { Sostenibilidad } \\
\text { institucional }\end{array}$ & $\begin{array}{l}\text { Índice de capacidad operativa de } \\
\text { los gobiernos locales municipales }\end{array}$ & Táctico \\
\hline 13 & Financiero & Índice de dependencia fiscal & Estratégico \\
\hline 14 & Financiero & $\begin{array}{l}\text { Porcentaje de fondos no } \\
\text { reembolsables }\end{array}$ & Operativo \\
\hline 15 & Financiero & $\begin{array}{l}\text { Porcentaje de fondos } \\
\text { rembolsables de fuentes } \\
\text { nacionales e internacionales }\end{array}$ & Operativo \\
\hline
\end{tabular}

Fuente: Elaboración propia.

La población estuvo conformada por el universo o conjunto de personas que intervienen en el proceso de planificación del Gobierno Autónomo Descentralizado Municipal del cantón Portoviejo, responsables de la calidad de su plan de desarrollo y ordenamiento territorial.

Se aplicó un muestreo no probabilístico, de tipo intencional a criterio de los investigadores que dividieron la población en tres muestras de grupo:

Grupo 1: estuvo conformada por funcionarios públicos con cargos de especialistas y analistas identificados como delegados de planificación de las direcciones cantonales de desarrollo institucional, desarrollo territorial, desarrollo social y desarrollo económico, cuyo trabajo está vinculado con el sistema de planificación del GAD Portoviejo. Esto dio un total de 22 personas. 
Grupo 2: estuvo conformada por los servidores de la dirección cantonal de planificación para el desarrollo, dando un total de 13 personas.

Grupo 3: estuvo conformado por los directores cantonales de planificación para el desarrollo, desarrollo institucional, desarrollo territorial, desarrollo social y desarrollo económico, dando un total de 5 personas.

La tabla 2 muestra la aplicación de las técnicas e instrumentos a cada grupo poblacional.

Tabla 2.

Relación entre las técnicas e instrumentos aplicados

\begin{tabular}{lccll}
\hline Objetivo de la investigación & $\begin{array}{l}\text { Tipo de } \\
\text { investigación }\end{array}$ & Población & Técnicas & Instrumentos \\
\hline $\begin{array}{l}\text { Evaluar la efectividad del } \\
\text { sistema de planificación del } \\
\text { gobierno Autónomo }\end{array}$ & Documental & No aplica & $\begin{array}{l}\text { Análisis } \\
\text { documental }\end{array}$ & Fichas \\
$\begin{array}{l}\text { Descentralizado municipal del } \\
\text { cantón Portoviejo, reflejada en }\end{array}$ & De campo & Grupo 1 - 2 & Encuestas & Cuestionario de Likert \\
$\begin{array}{l}\text { la calidad de su Plan de } \\
\begin{array}{l}\text { Desarrollo y Ordenamiento } \\
\text { Territorial }\end{array}\end{array}$ & Grupo 3 & Entrevistas & $\begin{array}{l}\text { Libreta de notas y guía } \\
\text { de entrevistas }\end{array}$ \\
\hline
\end{tabular}

Fuente: Elaboración propia.

\section{Resultados}

El Estado central y la gestión pública estructurados bajo una lógica de planificación sectorial no han alcanzado el desafío de pasar a una planificación territorial por objetivos, enfocada en resultados, que privilegie la articulación con las localidades, a fin de construir una visión de política pública como un todo estructurado. En ese marco, se ha evidenciado que los lineamientos postpandemia para la actualización de los PDOT, emitidos por el órgano rector de la planificación, no han sido definidos para cada nivel de gobierno en correspondencia a sus competencias, sino que aborda ejes generales quedando a discreción de cada gobierno local su adaptación.

No se evidencia una propuesta estructurada desde la planificación nacional del ejecutivo que articule acciones de corto y mediano plazo en los territorios, en post de la recuperación sanitaria y económica por la que atraviesa el país en los actuales momentos.

Institucionalizar el proceso de planificación en los GAD, es de notada importancia, dado que de ese modo se convierte en un proceso sistémico y estructurado que garantiza el cumplimiento de las metas propuestas por el GAD. En ese sentido, la figura 1 muestra los pasos para institucionalizar el proceso de planificación y ordenamiento territorial de nivel cantonal, establecidos en la guía para la formulación/actualización del PDOT cantonal vigente, emitido por la Secretaría Técnica Planifica Ecuador.

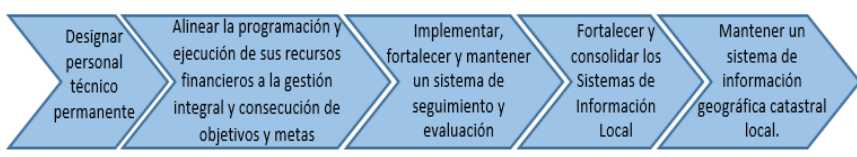

Figura 1. Principales pasos para la institucionalización de la planificación.

Fuente: Guía para la formulación/actualización del PDOT cantonal emitido por la Secretaría Técnica Planifica Ecuador (Secretaría Técnica Planifica Ecuador, 2019).

En el $100 \%$ de las encuestas aplicadas a los funcionarios del GAD Portoviejo se incorporan preguntas que abordan estos pasos, cuyos resultados evidencian que existe un equipo técnico que cumple los perfiles de los puestos requeridos, bajo la estructura de la Dirección Cantonal de
Planificación para el Desarrollo, en cuyo mapa de componentes consta como producto el PDOT, como lo muestra la figura 2.

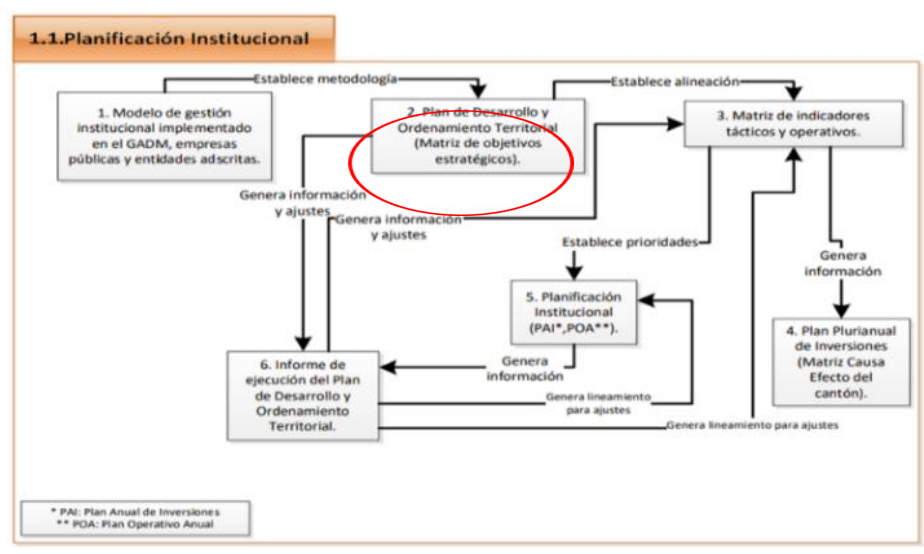

Figura 2. Mapa de componentes.

Fuente: Dirección Cantonal de Planificación. GADM Portoviejo.

Adicionalmente el GAD basa su gestión en un sistema de planificación bajo el enfoque de calidad, que determina la identificación y sistematización de procedimientos. Para el caso de los PDOT existen procedimientos vinculantes de la gestión de proyectos, administrativa/financiera y de control.

La Dirección Cantonal de Planificación tiene una estructura consolidada como se muestra en la figura 3 ; organizada de tal manera que cumple el ciclo PHVA en el sistema de planificación del GAD. Planificar y hacer que se desarrollen en la dirección de planificación estratégica y gestión de la calidad, verificar en la coordinación de control de gestión y actuar en la dirección de sostenibilidad, según la información de entrevista otorgada por el director cantonal de planificación.

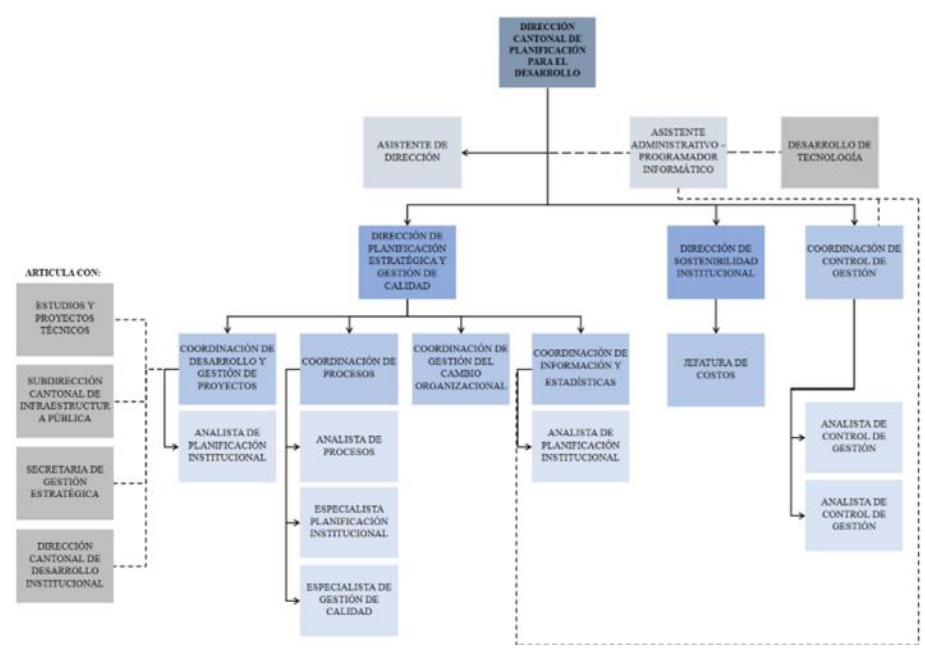

Figura 3. Orgánico de la Dirección Cantonal de Planificación. Fuente: Dirección Cantonal de Planificación. GADM Portoviejo.

El sistema de información local del GAD se encuentra en proceso de construcción, dado que el propio sistema nacional de información (SIN) no se encuentra actualizado. La consolidación de la información requiere permanente actualización, así como un sistema tecnológico que facilite el procesamiento de los datos para su interpretación.

Actualmente, la plataforma de intranet municipal se encuentra en funcionamiento, cuyo objetivo es facilitar el acceso de información generada por el GAD a los propios funcionarios y el flujo de

Planes de desarrollo y ordenamiento territorial. Caso de estudio: Gobierno Autónomo Descentralizado municipal de Portoviejo

Arteaga Demera, Pelegrín Entenza, Gómez García 
comunicación. En dicha plataforma se encuentra el modelo de gestión del GAD, que es una atribución del alcalde según el COOTAD, como se evidencia en la figura 4, que contiene entre otras cosas la propuesta de desarrollo para Portoviejo, reflejada en los planes de desarrollo, según lo mencionado en la entrevista realizada al director cantonal de planificación.

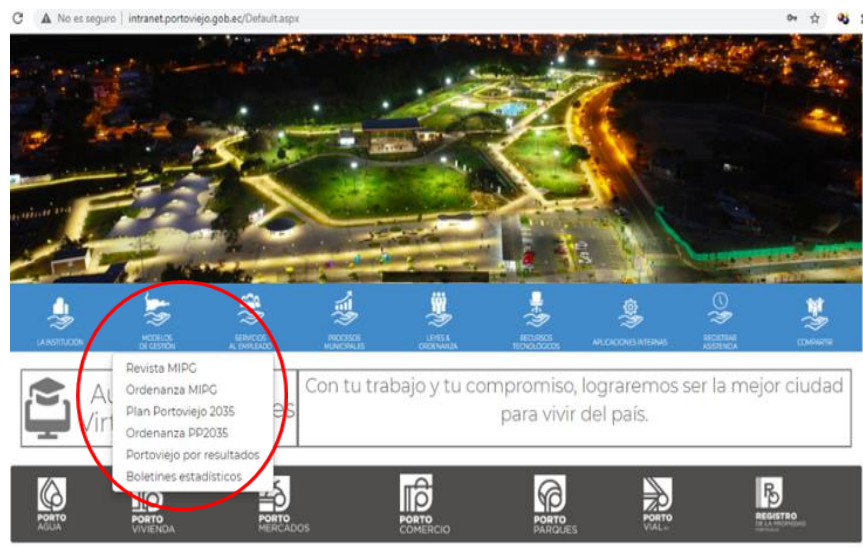

Figura 4. Intranet municipal GAD Portoviejo.

Fuente: GAD Portoviejo.

El $80 \%$ de los encuestados mencionan la existencia de un sistema de información catastral como una ventaja para la definición de las categorías de ordenamiento territorial del cantón Portoviejo, cuya cobertura como lo muestra la figura 5, supera el $80 \%$ en las zonas urbanas y rurales, información que es de mucha utilidad para la definición de programas zonificados en territorio urbano y rural, según lo mencionado en la entrevista al director cantonal de desarrollo territorial.

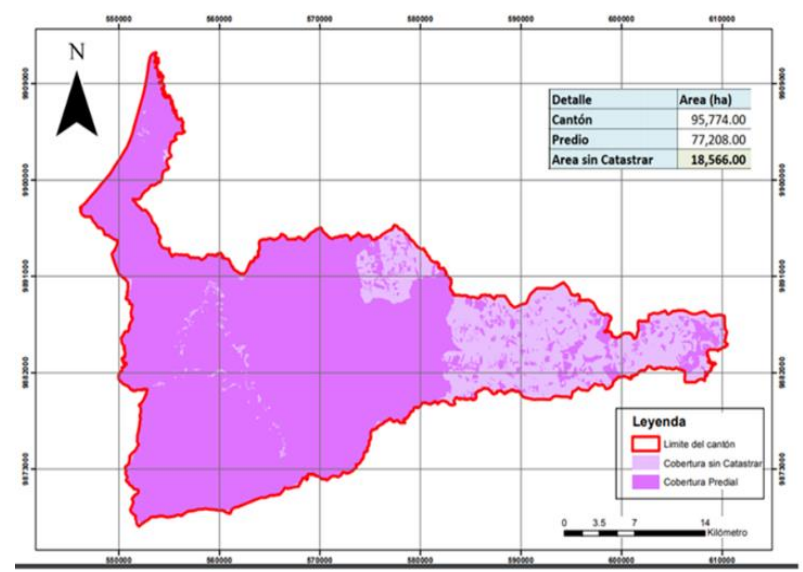

Figura 5. Mapa temático de cobertura predial en el cantón Portoviejo.

Fuente: Dirección de Información, Avalúos, Catastros y Permisos municipales (corte octubre del año 2020).

La Secretaría de Planificación a través del Índice de Cumplimiento de Metas (ICM) mide el cumplimiento efectivo de la meta de resultado de los planes de desarrollo y ordenamiento territorial en un período determinado. Se evidenció, como se expresa en la figura 6, que el gobierno local de Portoviejo ha cumplido hasta la fecha de apertura del sistema con los reportes correspondientes.

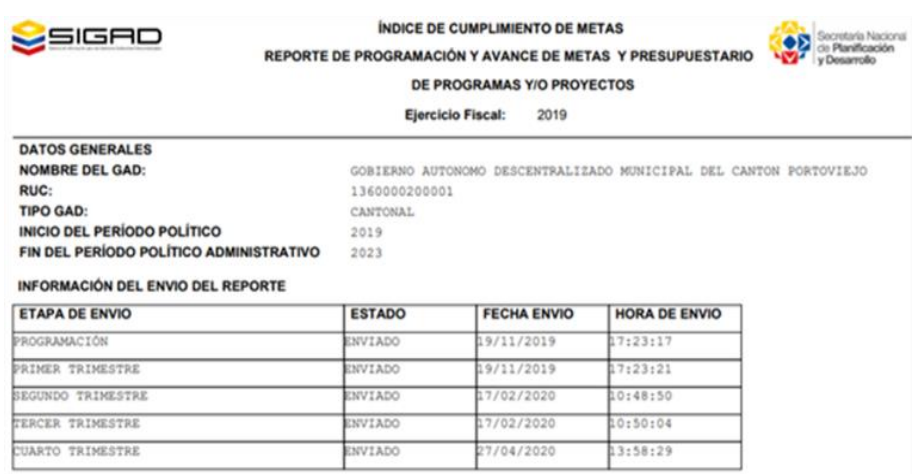

\section{INFORMACION DE LAS METAS, PROGRAMAS YIO PROYECTOS \\ NÚMERO DE METAS INGRESADAS: \\ NÚMERO DE PROGRAMAS YIO PROYECTOS INGRESADOS: \\ MONTO TOTAL DE PROYECTOS INGRESADOS: \\ MONTO TOTAL DE PROYECTOS EN EJECUCION:}

Figura 6. Reporte de ICM.

Fuente: Secretaría Técnica Planifica Ecuador. Módulo SIGAD.

En las entrevistas realizadas a los cinco directores cantonales de desarrollo territorial, social, institucional, económico, y de planificación, referente al seguimiento de las metas de los programas y proyectos del PDOT, estos manifestaron la puesta en marcha de un sistema informático denominado "Portoviejo por Resultados" que incorpora una metodología que aporta a la gestión de la información, como al registro, seguimiento y evaluación permanente de la gestión institucional.

Como se expresa en la figura 7 , el mencionado sistema parte del registro de la información, la que se procesa en función de la dinámica estratégica y operativa de las operaciones del GAD, a las que eventualmente se realiza el seguimiento y control para detectar de manera oportuna los riesgos que provocan atrasos o inmovilización en la operación, para finalmente evaluar los resultados y tomar los correctivos que permitan el cumplimiento de las metas planificadas.
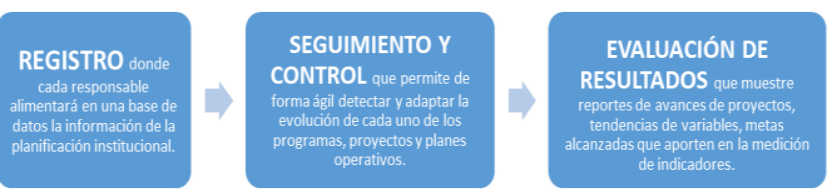

Figura 7. Ventajas del sistema Portoviejo por Resultados.

Fuente: Manual de acceso, uso y administración del sistema Portoviejo por Resultados. Módulo Operación.

La dependencia fiscal de los gobiernos locales es un indicador determinante para la solvencia financiera y su capacidad de endeudamiento, así lo señala en la entrevista el director cantonal de desarrollo institucional. Tradicionalmente ha sido causa para la inmovilización de los GAD en la generación del desarrollo, pues han optado por manejar administraciones tradicionales cuidadosas de los gastos corrientes y no han asumido el liderazgo de mejorar los ingresos propios bajo eficientes sistemas de manejo de los servicios públicos, y un adecuado manejo de la cartera municipal.

Se evidencia en la figura 8 un mejoramiento en el nivel de dependencia por las transferencias del gobierno central entre los años 2014 al 2018. Lo que el director cantonal de desarrollo institucional atribuye al mejoramiento en la recaudación de ingresos propios.

Para el año 2019 se evidencia un ligero crecimiento, al respecto, el director cantonal de planificación menciona que están trabajando en las 
estrategias para mejorar la recaudación tributaria y no tributaria del GAD, aunque producto de la pandemia han tenido una rebaja en la captación de los impuestos que alimentan los ingresos del GAD.

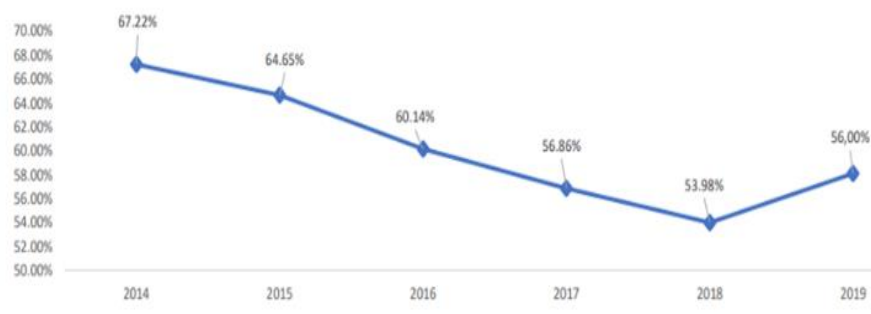

Figura 8. Índice de dependencia fiscal del GAD de Portoviejo.

Fuente: Diagnóstico estratégico del componente institucional del Plan Portoviejo 2035.

Es propicio mencionar que a partir de la información bibliográfica analizada, se evidencia que la Constitución plantea un nuevo modelo de Estado para fortalecer los poderes locales, recuperar el rol de la planificación; y define como características del mismo a la descentralización y a la desconcentración, en ese sentido, un elemento importante es el Modelo de Equidad Territorial en la Provisión de Bienes y Servicios Públicos, previsto en el Código Orgánico de Organización Territorial, Autonomía y Descentralización, basado en siete criterios constitucionales, como se muestran en la figura 9, y cuyo espíritu era eliminar las transferencias discrecionales y las prácticas clientelares, garantizando que las asignaciones a los gobiernos autónomos descentralizados sean determinadas de forma técnica y en base a las características propias de cada territorio.

\begin{tabular}{|l|c|c|c|}
\hline \multirow{2}{*}{\multicolumn{2}{|c|}{ Criterios de Distribución }} & \multicolumn{3}{c|}{ Nivel de Gobierno } \\
\cline { 2 - 4 } & Provincia & Cantón & Parroquia \\
\hline Tamaño de la población & $10 \%$ & $10 \%$ & $15 \%$ \\
\hline Densidad poblacional & $14 \%$ & $13 \%$ & $15 \%$ \\
\hline Necesidades Básicas Insatisfechas & $53 \%$ & $50 \%$ & $50 \%$ \\
\hline Logros en mejoramiento de los niveles de vida & $5 \%$ & $5 \%$ & $5 \%$ \\
\hline Esfuerzo Fiscal* & $2 \%$ & $6 \%$ & $0 \%$ \\
\hline Estuerzo Administrativo & $6 \%$ & $6 \%$ & $5 \%$ \\
\hline Cumplimiento de metas & $10 \%$ & $10 \%$ & $10 \%$ \\
\hline
\end{tabular}

Figura 9. Modelo de equidad territorial.

Fuente: Código Orgánico de Organización Territorial, Autonomía y Descentralización COOTAD.

Derivados del análisis del modelo, y a juicio de los autores, los criterios de distribución son apropiados, dado que están orientados a la consolidación de un Estado cohesionado y policéntrico, que permitirá superar las desigualdades que existen en el territorio nacional, potenciado con un modelo de descentralización nacional que se inspira en los principios de solidaridad y equidad. Un estudio adicional merece el análisis de aplicación de los criterios y sus resultados, dado que se desconoce la información sobre el cálculo del modelo de equidad territorial desde el Ministerio de Finanzas.

El $100 \%$ de las encuestas y el $100 \%$ de las entrevistas mencionan conocer los objetivos estratégicos del modelo de desarrollo de Portoviejo.

En la entrevista al director cantonal de planificación, este señala que los objetivos estratégicos guardan relación con uno de los índices de corte internacional que mide la prosperidad de los territorios, teniendo en cuenta tanto ingresos como bienestar, aunque menciona que generalmente ha sido un indicador medido a nivel nacional, no ha sido aplicado a nivel local, y por tanto no se tienen datos de resultados al respecto.

La figura 10 evidencia los doce objetivos estratégicos que según lo señala en la entrevista el director cantonal de desarrollo territorial son medidos con eventualidad según el procedimiento definido en la fase de seguimiento y evaluación del PDOT.

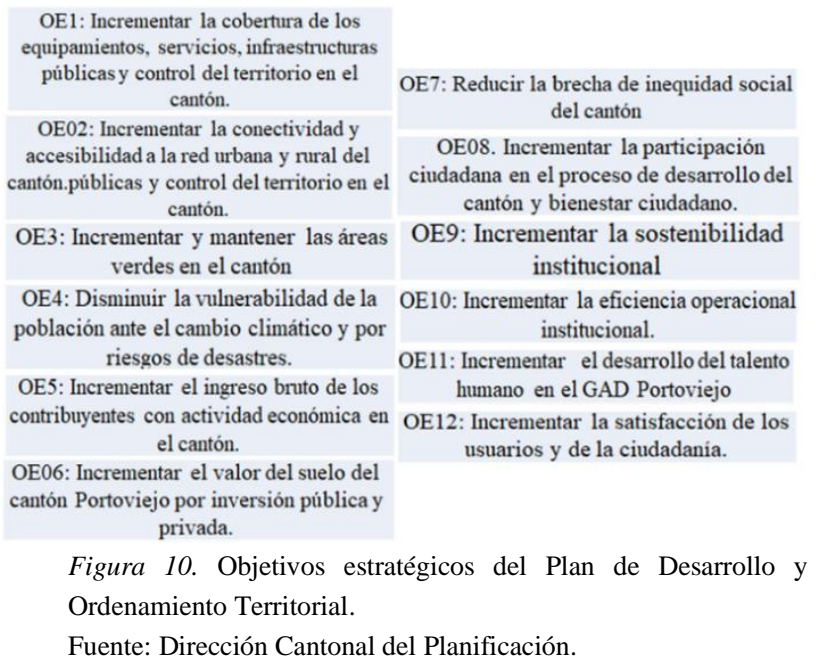

En la revisión bibliográfica se evidenció que según el índice de prosperidad Legatum 2019, Ecuador se encuentra en el No. 80 de entre 167 países evaluados, donde reside el $99,4 \%$ de la población mundial. En el año 2016, la Universidad FLACSO, con el auspicio de organismos internacionales, como ONU Hábitat y el Banco Interamericano de Desarrollo (BID), preparó el primer reporte del índice de prosperidad urbana, aplicado a 27 ciudades ecuatorianas. La investigación, utilizó información del censo nacional de 2010 y de la encuesta de calidad de vida de 2014, y consideró dimensiones, como productividad, desarrollo de infraestructura, calidad de vida, equidad e inclusión social, sustentabilidad ambiental, gobernanza y legislación.

Tras aplicar la metodología, se determinó que, según los estándares internacionales, las ciudades ecuatorianas se ubican en rangos de prosperidad moderadamente débil. Las conclusiones del estudio establecen que las tres ciudades mejor posicionadas son Cuenca, Ambato y Quito, las tres últimas en el estudio, en cambio, son Orellana, Morona y Lago Agrio, y Guayaquil, Portoviejo y Riobamba se encuentran en el medio de la tabla como se muestra en la figura 11.

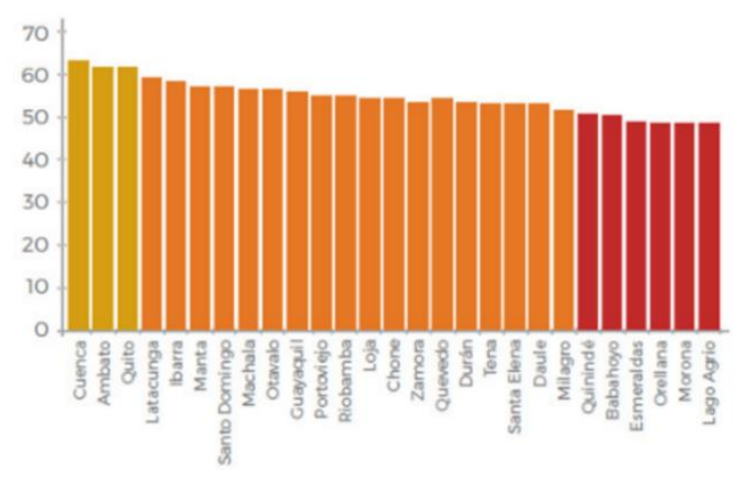

Figura 11. Medición del Índice de Prosperidad. Fuente: ONU Hábitat 2012. 
En el $100 \%$ de las entrevistas y encuestas realizadas se incluyeron varias preguntas sobre los principales problemas y las principales ventajas para la planificación de los PODT. Del análisis, procesamiento y sistematización de los datos, se evidencian en la figura 12 y 13 respectivamente los resultados declarados por los entrevistados y encuestados.

Se muestra en la figura 12 que los principales problemas en la planificación de los PDOT se dan por la ausencia de información a escala local, y por los lineamientos nacionales alejados de las realidades locales. Al respecto, el $100 \%$ de los participantes coinciden en que son los problemas medulares. El $88 \%$ señalan además que la disparidad en la territorialización de las políticas públicas del Estado Central son un problema, así como la ausencia de un sistema informático de seguimiento y evaluación del plan, del cual el 50\% de los participantes lo señalan como uno de los problemas principales.

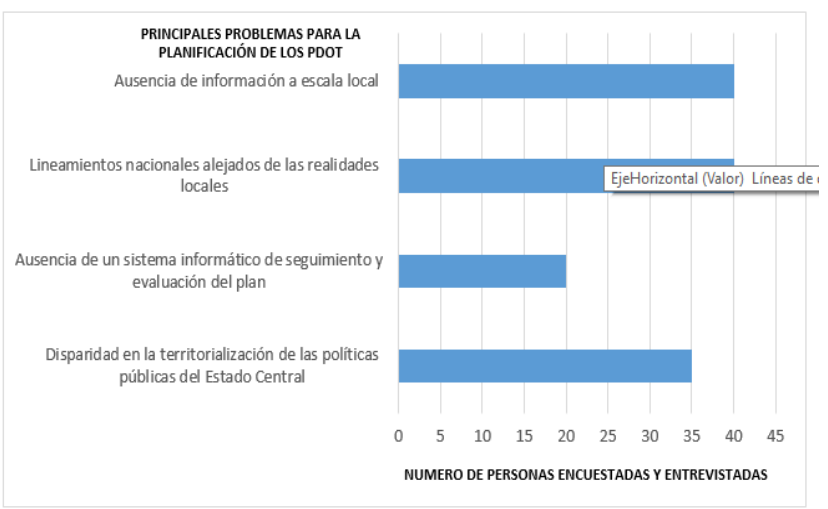

Figura 12. Principales problemas en la planificación de los PDOT.

Fuente: Elaboración propia.

Así también se sistematizaron las principales fortalezas para la planificación de los PDOT y se expresan de la siguiente manera (ver figura 13):

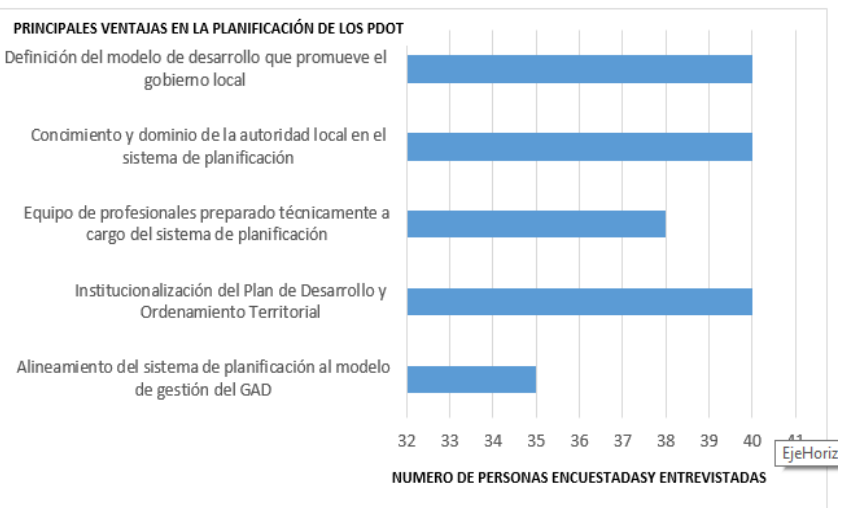

Figura 13. Principales fortalezas en la planificación de los PDOT.

Fuente: Elaboración propia.

El $100 \%$ de los encuestados y entrevistados mencionan que las principales fortalezas están relacionadas con el conocimiento y dominio de la autoridad local sobre el sistema de planificación, lo que ha facilitado la institucionalización de los planes de desarrollo y ordenamiento territorial donde se define el modelo desarrollo que promueve el gobierno local, a través de sus objetivos estratégicos. El 95\% de los participantes señala también como fortaleza el hecho de que exista un equipo de profesionales preparados para el proceso de planificación y finalmente el $88 \%$ mencionan que la aplicación de un modelo de gestión que alinee el sistema de planificación del GAD es también una de las principales ventajas.

Se evidenció que el GAD Portoviejo, bajo el enfoque de un modelo de gestión focalizado en la mejora continua, alcanzó el nivel de "Comprometido" del Programa del Modelo Ecuatoriano de Calidad y Excelencia liderado por el Ministerio de Trabajo.

Para los gobiernos locales queda el desafío de disrumpir prácticas tradicionales en la gestión, que no han dado resultados en el desarrollo de las localidades, a través de nuevos modelos del quehacer público, a partir del reconocimiento de las principales deficiencias del territorio para la transformación de las condiciones de vida de sus habitantes.

Los resultados de lo planificado frente a lo ejecutado determinan en gran medida la efectividad de la operación de los planes de desarrollo, pero no necesariamente garantizan la evolución del desarrollo de los territorios. Por esa razón, es sumamente importante que en el transcurso del proceso de planificación basado en modelos de calidad, se procure una reflexión crítica de integración entre cada uno de los sectores o sistemas, desde la validez del diagnóstico, pasando por el modelo territorial, hasta el eficiente e integral modelo de gestión vigilando siempre la mayor integración intersectorial posible que coadyuve a la consecución de los objetivos estratégicos hacia la mejora de vida de la población y de sus territorios.

\section{Discusión}

Se comprobó que el sistema de planificación del Gobierno Autónomo Descentralizado municipal del cantón Portoviejo es adecuado, según los estándares del Modelo Ecuatoriano de Calidad y Excelencia, lo que se muestra en los resultados de esta investigación.

El Gobierno Autónomo Descentralizado municipal del cantón Portoviejo ha adaptado su sistema de planificación a un modelo de corte internacional llamado Excutium Premiun, que persigue estándares de calidad.

El sistema de planificación le ha permitido al cantón organizar su gestión financiera, a través del desarrollo de un programa que otorga la responsabilidad a la directora financiera de reducir el índice de dependencia fiscal, a través de estrategias de recaudación oportuna.

El sistema de planificación, expresado principalmente en el PDOT como máximo instrumento de la planificación local, se encuentra institucionalizado, en función de lo establecido por la Secretaria Técnica de Planificación.

El conocimiento y dominio de la gestión de planificación por parte de la autoridad municipal ha reforzado el ciclo de la planificación en la institución, garantizado una visión estratégica del desarrollo a largo plazo como bien lo señala Máttar \& Perrotti (2014).

El sistema de información catastral de Portoviejo es de los más avanzados a nivel nacional, un hecho importante, porque facilita la planificación urbana y rural.

La planificación operativa se encuentra alineada a la planificación estratégica, en función del modelo de gestión y planificación del GAD, un modelo basado en la mejora continua, en concordancia con lo planteado por Colorado (2009), buscando garantizar niveles de eficiencia y 
oportunidad en la dotación de servicios públicos en la generación de política públicas.

A nivel nacional, es necesario institucionalizar la planificación estratégica local y maximizar las estrategias necesarias para lograr y sostener la articulación con la planificación nacional, y en buena medida se podrá lograr a través de un sistema de seguimiento y control eficiente entre lo que se planifica y ejecuta, como lo está desarrollando el GAD con su sistema Portoviejo por Resultados.

Para el GAD Portoviejo, los PDOT no son un proceso meramente normativo, están direccionados a la transformación territorial y al desarrollo, a través de la motivación de organismos multilaterales para invertir en Portoviejo. Es un instrumento que genera valor añadido y promueve la competitividad local.

La planificación del desarrollo local debe estar focalizada a las transformaciones territoriales bajo condiciones de sostenibilidad, eficacia y legitimidad, derivado de la acción colectiva y territorializada de sus pobladores que son corresponsables del progreso y bienestar de sus territorios, en esa medida, uno de los objetivos estratégicos del GAD es incrementar la participación ciudadana en el proceso de desarrollo del cantón.

En términos generales, la evidencia del GAD de Portoviejo sobre la institucionalización de los planes de desarrollo y ordenamiento territorial, mediante la definición de un equipo de trabajo que permanentemente realice seguimiento y control al plan y se evalúen los avances de las metas, podría ser un modelo aplicable en otros gobiernos locales.

Planificar el desarrollo de las ciudades no es una tarea sencilla, parte de la aplicación del marco jurídico nacional y local establecido. En Ecuador, en estos últimos años se han hecho esfuerzos importantes para dotar a las diferentes organizaciones territoriales: gobiernos autónomos descentralizados del nivel regional, provincial, cantonal y parroquial, de planes de desarrollo y ordenación territorial, con el afán de evidenciar las necesidades prioritarias y las fortalezas de cada localidad.

Dejar de planificar y ordenar desde un enfoque meramente arquitectónico, es el desafío de los gobiernos locales, pues no basta una "obra bonita", es necesario la reconfiguración de las ciudades desde la perspectiva humana, como lo señala Max-Neef et al. (2010), donde los equipamientos urbanos y rurales estén destinados al uso de las personas, en ese sentido los PDOT son el instrumento para hacer de las localidades, espacios dignos de convivencia.

Seguir pensando que los PDOT son un simple documento que luego de su aprobación, -trámite administrativo que se realiza para evitar una sanción de la Súper Intendencia de Ordenamiento Territorial- no se vuelve a saber de él; es un desacierto, es necesario que los gobiernos locales asuman con responsabilidad la primera competencia determinada en el Código Orgánico de Ordenamiento Territorial Autonomía y Descentralización COOTAD, que habla sobre la planificación del desarrollo local.

Pese a los avances importantes que ha tenido el GAD Portoviejo en su técnica planificadora, presenta limitaciones, sobre todo provocadas por factores externos, relacionadas a la planificación nacional.

Es necesario fortalecer el sistema de información local, sobre todo en la generación de datos socioeconómicos del cantón y la disponibilidad de bienes y servicios públicos georreferenciados.

Desde el seguimiento y evaluación de la planificación, el Plan Nacional de Desarrollo está acompañado por un sistema de monitoreo y evaluación con el afán de conocer los impactos de la gestión pública y generar alertas oportunas para la toma de decisiones, a partir de medir lo programado frente a lo realizado (Secretaría Nacional de Planificación y Desarrollo, 2017).
Otra de las disparidades entre lo nacional y lo local es la territorialización de las metas de la planificación nacional, sigue aún lejana la posibilidad de valorar al territorio como el espacio donde se concretan todas las metas propuestas.

La territorialización de las políticas públicas desde el Estado Central está alejada de las verdaderas demandas de los territorios, los lineamientos son generalmente a escala nacional, sin considerar las particularidades de las localidades y sus capacidades institucionales.

En términos generales, es necesario para la ciencia reestructurar el sistema de conocimientos respecto a los planes de desarrollo para la organización de las localidades. La planificación del desarrollo va más allá de la definición de metas e indicadores, incluye un proceso responsable y organizado de la construcción de las ciudades a partir de las necesidades humanas corresponsablemente con el entorno y la naturaleza como lo enfoca UNESCO (2017).

Es imprescindible que los gobiernos locales en articulación con la academia desarrollen estudios técnicos de medición del índice de prosperidad local, definiendo un mínimo de indicadores de manejo universal y público, para efectos de construir políticas públicas basadas en objetivos estratégicos.

Urge un cambio de modelo, que rompa con las prácticas burocráticas del momento, que garantice alternativas de desarrollo, sin caer en la visión lineal y restringida del progreso. Los elevados niveles de pobreza son producto de la desigualdad extrema, es un problema de orden político y erradicarla debe ser para todos un imperativo moral, por eso se necesitan planes eficientes, coherentes y transparentes que demanden al Estado inversión pública de calidad y oportuna que apunte al logro de las metas propuestas en los territorios.

A propósito de los eventos emergentes por los que atraviesan los territorios, replantear la planificación y el ordenamiento territorial constituye una oportunidad para devolver a las personas el derecho a la ciudad, y como lo dice ONU (2018) los gobiernos locales tienen una importante participación y una amplia responsabilidad.

\section{Referencias bibliográficas}

Abramo et al. (2016). Ciudades populares en disputa: ¿Acceso a suelo urbano para todos?. Quito: Ediciones Abya-Yala. Disponible en: http://dspace.ups.edu.ec/handle/123456789/12829

Andía, V. W. (2016). Enfoque metodológico para los objetivos estratégicos en la planificación del sector público. Disponible en: https://core.ac.uk/download/pdf/304901144.pdf

Alburquerque, F. (2004). El enfoque del desarrollo económico local. Buenos Aires.

Alonso, O. \& Otero, A. (2020). Ensayo sobre el desarrollo local y rural. España: Andavira Editora.

Asamblea Nacional Constituyente de Ecuador. (2008). Constitución. Montecristi: Registro Oficial No. 449.

Asamblea Nacional de Ecuador. (2010a). Código Orgánico de Ordenamiento Territorial, Autonomía y Descentralización. Montecristi. Quito.

Asamblea Nacional del Ecuador. (2010b). Código Orgánico de Planificación y Finanzas Públicas. Quito.

Asamblea Nacional del Ecuador. (2016). Ley Orgánica de Ordenamiento Territorial, Uso y Gestión de Suelo. Quito: Registro Oficial No 790.

Asamblea Nacional del Ecuador. (2019). Reglamento a la Ley Orgánica de Ordenamiento Territorial Uso y Gestión de Suelo. Quito.

Planes de desarrollo y ordenamiento territorial. Caso de estudio: Gobierno Autónomo Descentralizado municipal de Portoviejo

Arteaga Demera, Pelegrín Entenza, Gómez García 
Banco Mundial. (2020). Desarrollo Urbano.Whashington. Disponible en: https://www.bancomundial.org/es/topic/urbandevelopment/overvi ew

Bitar, S. (2016). Las tendencias mundiales y el futuro de América Latina. Edición 2016.

Blanco, H. (2003). Planeamiento del desarrollo local. Santiago de Chile: Naciones Unidas.

Brenes et al. (2014). "Arte x chepe”: Creatividad crítica en estudios de la ciudad. Disponible en: https://repositorio.iis.ucr.ac.cr/

Castellanos, C. E. Q. (2017). Gobernanza y teoría de las organizaciones. Revista Perfiles Latinoamericanos, 25(50), 39-57.

Castiella, L. (2016). La importancia de un modelo de planificación estratégica para el desarrollo de ciudades inteligentes. Buenos Aires.

Colorado, F. (2009). El ciclo PHVA de Deming y el proceso administrativo de Fayol. Academia. Recuperado de http://www. academia. edu

Consejo de Gobiernos Provinciales del Ecuador (CONGOPE). (2017). Alianzas público-privadas y desarrollo territorial. Quito: Ediciones Abya-Yala. Disponible en: https://biblio.flacsoandes.edu.ec/libros/digital/57078.pdf

Comisión Económica para América Latina y el Caribe (CEPAl). (2015). Panorama del desarrollo territorial en América Latina y el Caribe, 2015. Pactos para la igualdad territorial. Santiago de Chile: S.1500808 . Disponible

en: https://repositorio.cepal.org/bitstream/handle/11362/39223/1/S150 0808

Comisión Económica para América Latina y el Caribe (CEPAl). (2018). La agenda 2030 y los objetivos de desarrollo sostenible. Una oportunidad para América Latina y el Caribe. Santiago de Chile: Disponible https://repositorio.cepal.org/bitstream/handle/11362/40155/24/S18 01141_es.pdf

Gardner et al. (2016).Ciudades sostenibles: Del sueño a la acción: la situación del mundo 2016: Informe anual de Worldwatch Institute. Madrid.

Gehl, J. (2014). Ciudades para la gente. Buenos Aires: Ediciones infinito.

Gómez, C. (2008). El territorio como reivindicación de la comunidad rural. Argentina: Universidad Nacional de la Plata. Ponencia en $V$ Jornadas de Sociología de la UNLP. Disponible en: http://www.memoria.fahce.unlp.edu.ar/trab_eventos/ev.6101/ev.6 101.pdf

Gómez, M. J. A. (2015). Guía para la aplicación de UNE-EN ISO 9001: 2015. AENOR. España.

Gómez, L. I. (2020). Desarrollo Sostenible. España: Editorial Elearning. Gudynas, E. (2018). Nuevas coyunturas entre extrativismo y desarrollo. Montevideo: Centro Latinoamericano de Ecología Social.

Güell, J. M. F. (2019). Planificación estratégica de ciudades: Nuevos instrumentos y procesos (Vol. 10).

Máttar, J. \& Perrotti, D. (2014). Planificación, prospectiva y gestión pública. Reflexiones para la agenda de desarrollo. Santiago de Chile: CEPAL.

Max-Neef et al. (2010). Desarrollo a escala humana. Opciones para el futuro. Madrid: Biblioteca $\mathrm{CF}+\mathrm{S}$. Disponible en: http://habitat.aq.upm.es

Ministerio de Trabajo. (2018a). Modelo Ecuatoriano de Calidad y Excelencia. Ecuador.
Ministerio de Trabajo. (2018b). Guía metodológica de aplicación del Modelo Ecuatoriano de Calidad y Excelencia. Ecuador.

Molano, C. F. (2016). El derecho a la ciudad: de Henri Lefevbre a los análisis sobre la ciudad capitalista contemporánea. Bogotá: Universidad Pedagógica Nacional. Disponible en: https://www.redalyc.org/pdf/3459/345945922001.pdf

Noguera, T. J. (2016). La visión territorial y sostenible del desarrollo local. España: Universidad de Valencia. ISBN: 978-84-370-99316

Organización de las Naciones Unidas (ONU). (2018). Las ciudades seguirán creciendo, sobre todo en los países en desarrollo. Nueva York. Disponible en: https://www.un.org/development/desa/es/news/population/2018world-urbanization-prospects.html

Organización de las Naciones Unidas para la Educación, la Ciencia, y la Cultura (UNESCO). (2017). Educación para los objetivos de desarrollo sostenible. Francia.

Quispe, F. G. M. (2016). Visiones del desarrollo endógeno desde las comunidades locales. Revista Perspectivas, (37), 95-122.

RAE. (2017). Real Academia Española. Diccionario Usual. Recuperado de https://goo.gl/10AfIp.

Riffo, L. (2017). Desigualdades territoriales en América Latina. Santiago de Chile. Disponible en: https://www.cepal.org/sites/default/files/presentations/luis_riffo.p df

Romero, M. \& Vidal, V. (2018). Planificación estratégica territorial y alteraciones del plan general. Revista ciudad y territorios, estudios territoriales. Págs. 7-20.

Secretaría Nacional de Planificación y Desarrollo. (2017). Plan Nacional de Desarrollo: Toda una vida. Ecuador: Resolución No. CNP-0032017.

Secretaría Técnica Planifica Ecuador. (2019). Guía para formulación/ actualización del Plan de Desarrollo y Ordenamiento Territorial (PDOT) Cantonal. Quito. Disponible en: https://www.planificacion.gob.ec/wpcontent/uploads/downloads/2019/08/GUIA-CANTONAL-FINAL.pdf

Sen, A. (2000). El desarrollo como libertad. México: Gaceta Ecológica. Disponible en: https://www.redalyc.org/articulo.oa?id=53905501

Siles, R. \& Mondelo, E. (2018). Herramientas y técnicas para la gestión de proyectos de desarrollo PM4R. Banco Interamericano de Desarrollo. 